\title{
The Prevalence of Depression Among Nurses in Iran: A Systematic Review and Meta-Analysis
}

\author{
Zhila Fereidouni ${ }^{1}$, Azizallah Dehghan ${ }^{2}$, Majid Najafi Kalyani ${ }^{*}$ \\ ${ }^{1}$ School of Nursing, Fasa University of Medical Sciences, Fasa, Iran \\ ${ }^{2}$ Noncommunicable Diseases Research Center, Fasa University of Medical Sciences, Fasa, Iran \\ ${ }^{3}$ Department of Medical-Surgical Nursing, School of Nursing and Midwifery, Shiraz University of Medical Sciences, Shiraz, Iran
}

Corresponding Author: Majid Najafi Kalyani, PhD, Assistant Professor, Department of Medical-Surgical Nursing, School of Nursing and Midwifery, Shiraz University of Medical Sciences, Shiraz, Iran. Tel: +98-9363114570, Email: majidnajafi5@yahoo.com

Received September 2, 2018; Accepted October 13, 2018; Online Published December 27, 2018

\begin{abstract}
Introduction: Nurses are at risk of depression at a rate twice as high as other professionals. Depression in addition to individual effects also influence quality of work and organizational performance. Surveying the prevalence of depression in nurses will help policymakers make systematic plans in this regard. Because of the importance of this issue, the current systematic review and meta-analysis was conducted to determine the prevalence of depression among nurses in Iran.

Methods: All published studies concerning the prevalence of depression among nurses in Iran during the 20-year period of 1997 to 2017 were extracted and studied using the Preferred Reporting Items for Systematic Reviews and Meta-Analyses (PRISMA) guidelines. Searches were conducted for articles using the keywords "Nurses", "Depression", and "Iran" in the local databases of SID, Iran Medex, and Mag Iran and in the international databases of PubMed, Science Direct, CINAHL, and Google Scholar. The extracted data was analyzed using STATA software, version 11, using the random effects model.

Results: A total of 14 studies with 4062 participating nurses from different wards and hospitals were analyzed. The overall pooled estimate of depression prevalence among Iranian nurses was $26.88 \%$ (95\% Cl: $21.45 \%, 31.91 \%$ ). Subgroup analysis showed that the estimated prevalence rates of depression among military nurses and public nurses were $22.38 \%$ (95\% Cl: $19.45 \%, 25.30 \%)$ and $28.12 \%(95 \% \mathrm{Cl}$ : $21.46 \%, 34.78 \%)$, respectively.

Conclusions: The results of this meta-analysis revealed that the prevalence of depression among Iranian nurses was relatively high. Therefore, policymakers must pay more attention to planning and the prevention of depression in nurses.

Keywords: Nurse, Depression, Systematic Review, Meta-Analysis

Citation: Mo. The prevalence of depression among nurses in Iran: a systematic review and meta-analysis. Int J Med Rev. 2018;5(4):163-167. doi:10.29252/JJMR-050407.
\end{abstract}

\section{Introduction}

Mental disorders are a primary cause of the total burden of disability and morbidity worldwide. One common mental disorder is depression, which is estimated to affect $15 \%$ of adults. ${ }^{1}$ According to the World Health Organization (WHO), depression will become the first cause of disability-adjusted life years by $2030 .^{2}$ It is also expected that more than 350 million people will suffer from depression. ${ }^{2}$ The Center for Disease Control and Prevention (CDC) reported that for the period 2009-2012, 7.6\% of Americans aged 12 and over had depression. $^{3}$

Depression is defined as a disorder affecting the whole body and afflicting the person with mental, physical, social, and spiritual problems. ${ }^{4}$ Depression symptoms include low mood, feelings of worthlessness, suicidal thoughts and actions, and changes in the body's natural patterns (e.g., insomnia and malnutrition). ${ }^{5}$ Depression also negatively affects interpersonal relationships, the ability to make decisions and judge correctly, and the quality of life.,7

Nurses, as key members of the treatment team, experience a high level of stress daily due to the nature of their profession. ${ }^{8,9}$ Studies have shown a significant relationship between stress and depression symptoms in nurses. Nursing is a stressful profession that can cause depression. ${ }^{2}$ Nurses are twice as likely to suffer from depression for their professional life. ${ }^{2}$ Some major factors which cause depression in nurses are long work shifts, fatigue, and great stress. ${ }^{10}$ Studies have also indicated that depression makes nurses vulnerable in relationships and causes job dissatisfaction, job burnout, and early retirement. ${ }^{11,12}$ A nurse's mental health is a crucial factor to consider in human resources retention programs. Nurses are the largest group of employees in a healthcare system, and they are exposed to a considerable level of occupational stress. ${ }^{10}$

Copyright $\odot 2018$ The Author(s). This is an open-access article distributed under the terms of the Creative Commons Attribution License (http:// creativecommons.org/licenses/by/4.0), which permits unrestricted use, distribution, and reproduction in any medium, provided the original work is properly cited. 
Workplace conditions, type of relationships, and organizational culture affect the mental health of all personnel. ${ }^{13}$ Nurses experience not only occupational stress, but also increasing pressure due to the necessity of providing care services in critical situations. They are thus prone to both stress and depression in their highly stressful workplaces. ${ }^{14,15}$

The fact that nurses are regarded as a crucial factor in systems of healthcare and health promotion requires the development of programs to improve their mental health based on the reported statistical data on the prevalence of depression among nurses. Due to the importance of the nurses' mental health and its impact on the quality of care services, the present study aimed to evaluate the prevalence of depression among Iranian nurses through a systematic review and meta-analysis.

\section{Methods}

This systematic review and meta-analysis analyzed all the related Persian and English articles published by both foreign and domestic journals during 1997-2017, based on the Preferred Reporting Items for Systematic Reviews and MetaAnalyses (PRISMA) guidelines (Figure 1) in order to evaluate the prevalence of depression among Iranian nurses.

\section{Search Strategy and Eligibility Criteria}

The main question of the researcher in this systematic review and meta-analysis was, "What is the prevalence of depression among Iranian nurses?" To answer this question, all related published articles on depression among Iranian nurses were searched using the keywords "Depression", "Nurses", and "Iran" both separately and in combination in local (SID, IranMedex, and MagIran) and international databases (Google Scholar, CINAHL, PubMed, and Science Direct).

The study's inclusion criteria for articles were access to the article's full text, Persian or English language articles, and articles reporting on the prevalence of depression among nurses. Accordingly, those articles lacking the full text or reporting merely on depression mean scores were excluded.

\section{Data Collection and Extraction}

After data collection and summarization were completed, the recorded data included year of publication, location, sampling method, depression measurement tool, sample size, and prevalence of depression. In the first step, a total of 643 articles were collected by searching the mentioned local and international databases. In the next step, the number of articles fell to 127 after duplicate and overlapping articles were excluded according to their title and abstract. Then, 113 articles were also excluded according to the criteria and the complete review. Finally, 14 articles (12 Persian and 2 English) were included in the final analysis (Table 1).

\section{Data Analysis}

The statistical software STATA version 11 was employed for analyzing the extracted data. The $\mathrm{I}^{2}$-heterogeneity test and the Random Effect Model were also used to measure the heterogeneity of the data.

\section{Ethical Considerations}

This study was approved by the Ethics Committee of Fasa University of Medical Sciences (IR.FUMS.REC.1397.137). It should also be noted all related ethical aspects, including intellectual property rights and accurate referencing, were considered in this study in accordance with the criteria of the Ethics Committee.

\section{Results}

The present study analyzed 14 articles published by Persian and English language journals and covered a total of 4062 nurses working in public, private, and military hospitals. In

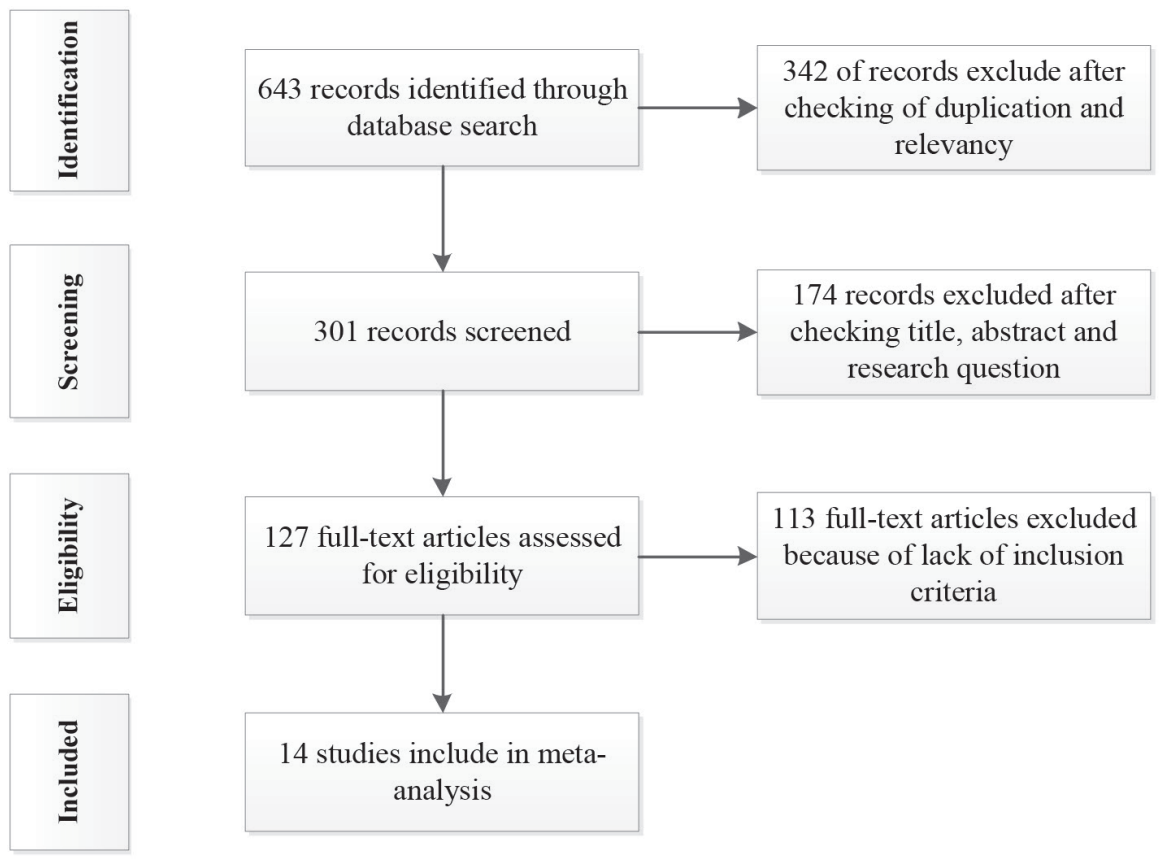

Figure 1. Diagram of the Study Protocol. 
Table 1. Summarized Findings of Studies Included in the Final Analysis

\begin{tabular}{|c|c|c|c|c|c|c|}
\hline Author(s) & Year & City & Sampling Method & Measurement Tool & Sample Size & Prevalence of Depression ( $\%$ ) \\
\hline Taghva et al & 2012 & Tehran & Random sampling & BDI & 173 military nurses & 20.2 \\
\hline Kazemi et al & 2010 & Tehran & Convenience sampling & BDI & 335 military nurses & 21.7 \\
\hline Asadzandi et al & 2008-2009 & Tehran & Multistage sampling & DASS-21 & 272 military nurses & 24.9 \\
\hline Khalilzadeh et al & 2005 & Urmia & Random sampling & BDI & 200 public nurses & 35 \\
\hline Yasemi et al & 2012 & Ilam & Census & BDI & 60 public nurses & 62.5 \\
\hline Khamseh et al & 2009 & Tehran & Random sampling & DASS-21 & 413 public nurses & 25.8 \\
\hline Khani et al & 2013 & Neyshabur & Random sampling & BDI & 196 public nurses & 26 \\
\hline Alipoor et al & 2014 & Fasa & Random sampling & DASS-21 & 142 public nurses & 32 \\
\hline Kasani et al & 2011 & Ilam & Non-Random sampling & BDI & 191 public nurses & 34 \\
\hline Khajeh Nasiri et al & 2000 & Tehran & Random sampling & BDI & 130 public nurses & 26.9 \\
\hline Mahmoudi et al & 2008-2009 & Sanandaj & Census & BDI & 314 public nurses & 21.5 \\
\hline Ardekani et al & 2007 & Shiraz & Proportional sampling & DASS-21 & 1195 public nurses & 11.2 \\
\hline Kavari & 2006 & Shiraz & Proportional sampling & BDI & 130 public nurses & 26.9 \\
\hline
\end{tabular}

terms of geographical distribution, most of the studies $(n=5)$ had focused on nurses in Tehran (Table 1) with the age range of 30-40 years. Prevalence rates of 11.2-62.5\% for depression among Iranian nurses were reported in 14 articles. Taghva et $\mathrm{al}^{12}$ included the participation of 173 military nurses in a survey based on the Beck Depression Inventory (BDI) in Tehran and reported a $20.2 \%$ prevalence of depression. Kazemi et $\mathrm{al}^{13}$ reported a $21.7 \%$ prevalence of depression among 335 military nurses in Tehran using BDI. Asad-zandi et $\mathrm{al}^{14}$ also reported a $24.9 \%$ prevalence among 272 military nurses in Tehran based on the Depression, Anxiety and Stress Scale (DASS-21).

Khalilzadeh et $\mathrm{al}^{15}$ reported a $35 \%$ prevalence among nurses in Urmia using BDI. The highest prevalence rate of depression (62.5\%) was reported by Yasemi et $\mathrm{al}^{16}$ in their study which covered 60 nurses in Ilam based on BDI. Khamseh et $\mathrm{al}^{8}$ reported a $25.8 \%$ prevalence among 413 nurses in Tehran using DASS-21. Khani et al ${ }^{17}$ also reported a $26 \%$ prevalence among 196 nurses in Neyshabur using BDI. A prevalence of depression of 32\% was reported among 142 nurses in Fasa using DASS-21. ${ }^{18}$ A study on 191 nurses in Ilam using BDI showed a $34 \%$ prevalence of depression. ${ }^{7}$ Khajeh Nasiri found a $26.9 \%$ prevalence in 130 nurses in Tehran using BDI. ${ }^{19}$ Mahmoudi et $\mathrm{al}^{20}$ concluded that $21.5 \%$ of nurses in Sanandaj were depressed through a survey of 314 nurses using BDI. Dehghani et $\mathrm{al}^{21}$ indicated a $16.4 \%$ prevalence of depression among 311 nurses using the Zung Self-Rating Depression Scale (SDS). Ardekani et $\mathrm{al}^{22}$ reported the lowest prevalence of depression (11.2\%) in nurses in Shiraz through a survey of 1,195 nurses using DASS-21, while Kavari had previously reported a $26.9 \%$ prevalence among 130 nurses in Shiraz. ${ }^{23}$

The prevalence of depression among Iranian nurses with a sample size of 4062 was estimated to be at $26.68 \%$ (95\% CI: 21.45-31.91) using the random effect model (Figure 2). To use this model, the main assumption was that the existing differences in the findings of different studies can be attributed to different sample sizes and prevalence rates. Heterogeneity was calculated to be $93.7 \%$.

Further analysis was carried out based on type of hospitals that were the workplaces of nurses. Subgroup analysis showed that the estimated prevalence rates of depression among military nurses and pubic nurses were 22.38\% (95\% CI: $19.45 \%, 25.30 \%$ ) (Figure 3) and 28.12\% (95\% CI: $21.46 \%$, $34.78 \%$ ) (Figure 4), respectively.

\section{Discussion}

Depression is one of the most common mental health disorders and is capable of negatively affecting a person's bodily functions and personal/professional life quality. ${ }^{1,5-7}$ The general prevalence of depression among Iranian nurses was estimated to be $26.68 \%$ (CI: 21.45-31.91). The present systematic review and meta-analysis discovered a relatively high prevalence of depression among Iranian nurses, which

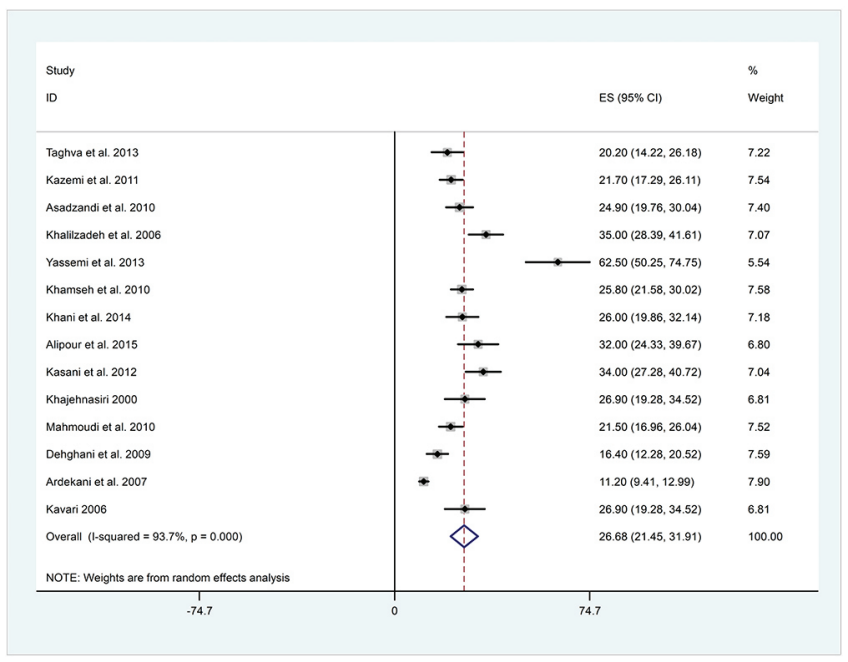

Figure 2. Prevalence of Depression Among Iranian Nurses Based on the Reviewed Studies. Horizontal lines around the main mean show a confidence interval of $95 \%$ for each study. The dotted line in the middle indicates the estimated total prevalence, and the lozenge shows the confidence interval of the total prevalence of depression. 


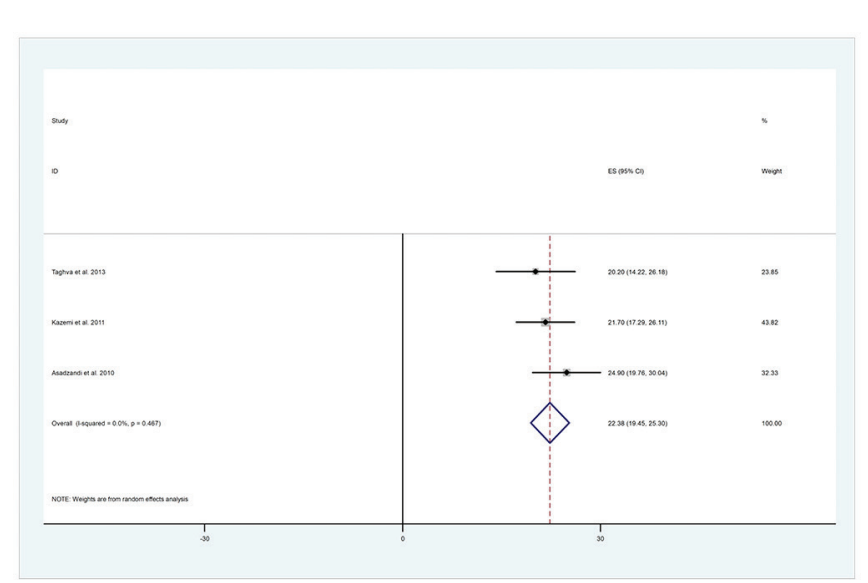

Figure 3. Prevalence of Depression Among Military Nurses Based on the Reviewed Studies. Horizontal lines around the main mean show a confidence interval of $95 \%$ for each study. The dotted line in the middle indicates the estimated total prevalence, and the lozenge shows the confidence interval of the total prevalence of depression.

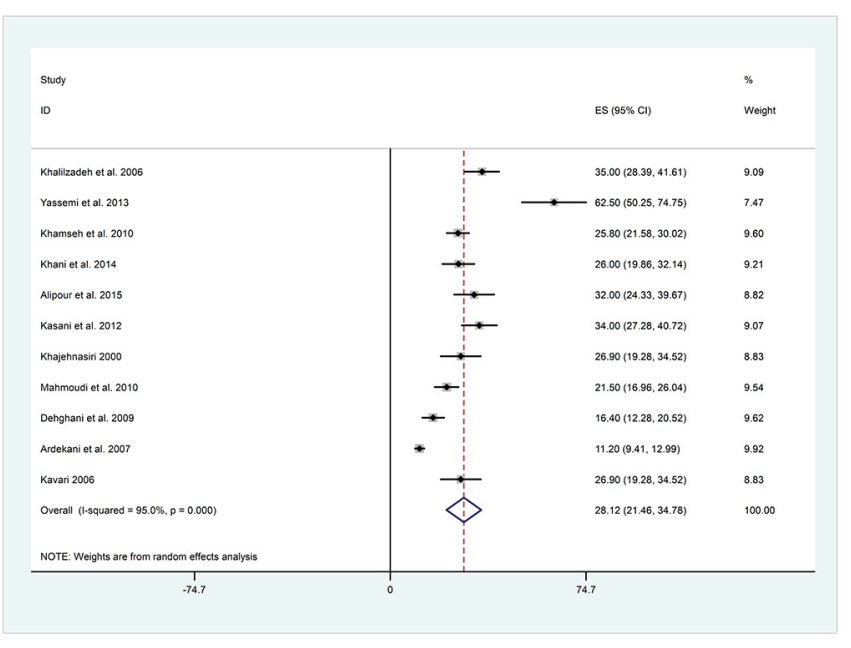

Figure 4. Prevalence of Depression Among Public Nurses Based on the Reviewed Studies. Horizontal lines around the main mean show a confidence interval of $95 \%$ for each study. The dotted line in the middle indicates the estimated total prevalence, and the lozenge shows the confidence interval of the total prevalence of depression.

had been previously reported to range $11.2 \%-62.5 \%$. This finding is fairly consistent with the prevalence of depression among the general population in Iran. Montazeri et $\mathrm{al}^{24}$ concluded that the prevalence of depression varies from $5.69 \%$ to $73 \%$ in Iran, depending on the study population. According to the WHO, the global prevalence of depression in nurses is estimated to range $15 \%-30 \% .^{14}$ The findings regarding the prevalence of depression among military nurses is consistent with the WHO's estimate, whereas the reported rate was above $30 \%$ among public nurses. ${ }^{7,13,16,18}$ The difference may be attributed to several factors such as personality traits and workplace conditions in addition to social factors.

The remarkable finding in this analysis is the lower estimated prevalence of depression among military nurses as compared with public nurses. This difference may possibly be attributed to the choice of sampling method. With the highest reported rate, Yasemi et al ${ }^{16}$ considered a sample size of 60 and employed the non-random sampling method. Their reported prevalence is somewhat closer to the average rates in those studies using the random sampling method with a larger sample size. Nonetheless, this difference requires further research and additional comparative studies on both military and public nurses.

According to the reviewed studies, females accounted for more than $50 \%$ of the studies' populations. ${ }^{11,12,14,18}$ The literature expectedly represented a higher prevalence of depression among females, since nurses are mostly female. Accordingly, female nurses are twice as likely to suffer from depression in the United States. ${ }^{2}$ The growing prevalence of depression in nurses, especially female ones, is attributed to factors like considerable workplace and home stressors for females. ${ }^{25}$ The researchers highlighted the role of other factors in the incidence of depression among nurses and argued that those who enjoy stronger social and familial support when facing problems and challenges are less likely to experience depression. ${ }^{2}$ Education was also reported as a major contributory factor to depression. Asadzandi et $\mathrm{al}^{14}$ demonstrated a relationship between education level and depression.

The literature further revealed a significant relationship between job satisfaction and depression in nurses, indicating that the lower the satisfaction is, the more likely the incidence of depression will be. ${ }^{26-28}$ The focus on organizational factors in the incidence and improvement of depression among nurses plays a key role in reducing the prevalence of depression. ${ }^{29}$ Those nurses who do not feel they are effective in their workplace and those whose work requires no diversity or creativity are more prone to depression. ${ }^{28}$ Letvak et $\mathrm{al}^{28}$ showed that workplace is a strong predictor of the incidence of depression in nurses and also suggested that improved working conditions could promote mental health and reduce depression.

Given the relatively high prevalence of depression among nurses and the importance of their mental health, it is necessary to identify those nurses prone to depression and thus minimize the prevalence of this disorder through the development of special programs and effectively protective interventions with the ultimate aim of improving the quality of care services delivered to patients. Depression screening for nurses should start from studying in nursing, so that susceptible individuals can be identified and the progress of the disease can be prevented..$^{30}$ Furthermore, the presence of mental health counselors in the nursing workplace plays a vital role in the prevention of depression. ${ }^{28}$

The study results recommend adopting a combined approach that includes both clinical tests and depression measurement tools in future research. In addition, it is imperative for health policy-makers to devise and adopt those strategies required for early diagnosis, treatment, and followup of depression among nurses.

\section{Conclusions}

According to the present systematic review and metaanalysis, the prevalence of depression among Iranian nurses 
is estimated to be $26.68 \%$. This considerable rate requires conducting supportive interventions for the prevention and early diagnosis of depression. It also necessitates providing a healthy and hygienic workplace for nurses where they feel safe and comfortable in order to improve the quality of care services.

\section{Authors' Contributions}

ZF was involved in the conception and design of the study, database search, early drafting of the manuscript, and revising the manuscript; $\mathrm{AD}$ acted as advisor in all stages of the research and statistical analysis; MNK contributed to the conception and design of the study, database search, extraction of data, and the writing and revision of the manuscript.

\section{Conflict of Interest Disclosures}

The authors declare they have no conflicts of interest.

\section{Funding/Support}

Fasa University of Medical Sciences, Fasa, Iran, supported this study, Grant Number: 96228.

\section{Acknowledgments}

This article was extracted from a research project approved by Fasa University of Medical Sciences, Fasa, Iran. The authors express their appreciation to the Deputy for Research of Fasa University of Medical Sciences for the support given to this project.

\section{References}

1. Lin HS, Probst JC, Hsu YC. Depression among female psychiatric nurses in southern Taiwan: main and moderating effects of job stress, coping behaviour and social support. J Clin Nurs. 2010;19(1516):2342-2354. doi:10.1111/j.1365-2702.2010.03216.x.

2. Brandford AA, Reed DB. Depression in Registered Nurses: A State of the Science. Workplace Health Saf. 2016;64(10):488-511. doi:10.1177/2165079916653415.

3. Centers for Disease Control and Prevention. Depression in the US household population, 2009-2012. National Center for Health Statistics, Division of Health Interview Statistics; 2014. https:// www.cdc.gov/nchs/data/databriefs/db172.htm. Accessed 2018.

4. Morrison-Valfre M. Foundations of Mental Health Care. Elsevier Health Sciences; 2016.

5. Blackmore ER, Stansfeld SA, Weller I, Munce S, Zagorski BM, Stewart DE. Major depressive episodes and work stress: results from a national population survey. Am J Public Health. 2007;97(11):2088-2093. doi:10.2105/ajph.2006.104406.

6. Melchior M, Caspi A, Milne BJ, Danese A, Poulton R, Moffitt TE. Work stress precipitates depression and anxiety in young, working women and men. Psychol Med. 2007;37(8):1119-1129. doi:10.1017/s0033291707000414.

7. Kassani A, Niazi M, Menati R, Alimohamadi Y, Menati W. Relationship between nurses' depression and quality of life: applying path analysis model. Quarterly Journal of Nersing Management. 2014;3(2):61-69. [Persian].

8. Khamseh F, Roohi H, Ebaady A, Hajiamini Z, Salimi H, Radfar S. Survey relationship between demographic factors and stress, anxiety and depression in nurses working in selected hospitals in Tehran city . Journal of Holistic Nursing and Midwifery. 2011;21(1):13-21. [Persian]

9. Chen YM, Chen SH, Tsai CY, Lo LY. Role stress and job satisfaction for nurse specialists. J Adv Nurs. 2007;59(5):497-509. doi:10.1111/ j.1365-2648.2007.04339.x.

10. Nasl Saraji G, Dargahi H. Study of quality of work life (QWL). Iran J Public Health. 2006;35(4):8-14.
11. Samadi S, Golmohammadi A, Seyedahmadian S, Rezapour T, Panahi $\mathrm{H}$. The relationship between job stress and occupational empowerment of nurses in selected military hospitals of the country. Journal of Police Medicine. 2013;2(1):31-37. [Persian].

12. Taghva A, Yazdani A, Ebrahimi MR, Alizadeh K, Sakhabakhsh M. Prevalence of depression in psychiatric nurses and comparison with other parts of the AJA hospitals. Nurse and Physician within War. 2013;23(24):11-16. [Persian].

13. Kazemi Galougahi MH, Kouhian K. A study of depression prevalence of nurses and it's effective factors in Tehran. Nurse and Physician Within War. 2010;14:8-11. [Persian].

14. Asad Zandi M, Sayari R, Ebadi A, Sanainasab H. Abundance of depression, anxiety and stress in militant Nurses. Journal of Military Medicine. 2011;13(2):103-108. [Persian].

15. Khalilzadeh R, Yavarian R, Khalkhali HR. The relationship of job stress, depression and anxiety of nursing staff of Urmia University of Medical Sciences. Journal of Urmia Nursing and Midwifery Faculty. 2005;3(1):10-17. [Persian].

16. Yasemi M, Peyman H, Khajavikhan J, et al. Prevalence of Depression among Nurses Working in the Operating Rooms and Intensive Care Units. Journal of Zabol University of Medical Sciences and Health Services. 2014;6(4):70-77. [Persian].

17. Khani H, Ghodsi H, Nezhadnik H, Teymori S, Ghodsi A. Depression and its relationship with hypochondriasis in nurses in Neyshabur, Iran. Military Caring Sciences. 2016;3(1):34-40. doi:10.18869/ acadpub.mcs.3.1.34. [Persian].

18. Alipoor R, Ebrahimi A, Omidi R, Hedayati A, Ranjbar H, Hosseinpour $\mathrm{S}$. Depression, anxiety, stress and related demographic variables in nurses of Valiasr hospital in Fasa University of Medical Sciences in 2014. Pajouhan Scientific Journal. 2015;13(4):51-59. [Persian].

19. Khajehnasiri F. A study of depression prevalence of nurses and its effective factors in Tehran Emam Khomeini Hospital. Tehran University Medical Journal. 2000;58(1):10-14. [Persian].

20. Mahmoudi S, Zehni K. The comparison of depression prevalence between shift work nurses in education hospitals of Kurdistan medical sciences university. Iranian Journal of Nursing Research. 2013;8(1):29-38. [Persian].

21. Dehghani M, Zoladl M, Boland-Parvaz S, Keshtkaran Z, Mahmoudi R, Jabbarnejad A. Asurvey on depression and its related factors in Nurses who work in Namazi Hospital of Shiraz University of Medical Sciences-2008. Iran Occupational Health Journal. 2009;6(3):29-34. [Persian].

22. Ardekani ZZ, Kakooei H, Ayattollahi SM, Choobineh A, Seraji GN. Prevalence of mental disorders among shift work hospital nurses in Shiraz, Iran. Pak J Biol Sci. 2008;11(12):1605-1609. doi:10.3923/ pjbs.2008.1605.1609.

23. Kavari SH. A study of depression prevalence in nurses and it's effect in Shiraz Namazi hospital. Middle East Journal of Family Medicine. 2006;4(3):17-21.

24. Montazeri A, Mousavi SJ, Omidvari S, Tavousi M, Hashemi A, Rostami T. Depression in Iran: a systematic review of the literature (2000-2010). Payesh. 2013;12(6):567-594. [Persian].

25. Bronder EC, Speight SL, Witherspoon KM, Thomas AJ. John Henryism, Depression, and Perceived Social Support in Black Women. J Black Psychol.2014;40(2):115-137.doi:10.1177/0095798412474466.

26. Schulz M, Damkroger A, Voltmer E, et al. Work-related behaviour and experience pattern in nurses: impact on physical and mental health. J Psychiatr Ment Health Nurs. 2011;18(5):411-417. doi:10.1111/j.1365-2850.2011.01691.x.

27. Mclntyre RS, Liauw S, Taylor VH. Depression in the workforce: the intermediary effect of medical comorbidity. J Affect Disord. 2011;128 Suppl 1:S29-36. doi:10.1016/s0165-0327(11)70006-4.

28. Letvak S, Ruhm CJ, McCoy T. Depression in hospital-employed nurses. Clin Nurse Spec. 2012;26(3):177-182. doi:10.1097/ NUR.0b013e3182503efo.

29. Campbell K, Hanna JC. Individual and Organizational Health and Well-Being. Pensacola, FL: American Association of Occupational Health Nurses; 2014.

30. Najafi Kalyani M, Jamshidi N, Salami J, Pourjam E. Investigation of the Relationship between Psychological Variables and Sleep Quality in Students of Medical Sciences. Depress Res Treat. 2017;2017:7143547. doi:10.1155/2017/7143547. 